\title{
Traditional health practitioner and the scientist: bridging the gap in contemporary health research in Tanzania
}

\author{
Z.H. MBWAMBO*, R.L.A. MAHUNNAH and E.J. KAYOMBO \\ Institute of Traditional Medicine, Muhimbili University College of Health Sciences, \\ P. O. Box 65001 Dar es Salaam, Tanzania
}

\begin{abstract}
Traditional health practitioners (THPs) and their role in traditional medicine health care system are worldwide acknowledged. Trend in the use of Traditional medicine (TRM) and Alternative or Complementary medicine (CAM) is increasing due to epidemics like HIV / AIDS, malaria, tuberculosis and other diseases like cancer. Despite the wide use of TRM, genuine concern from the public and scientists/biomedical heath practitioners (BHP) on efficacy, safety and quality of TRM has been raised. While appreciating and promoting the use of TRM, the World Health Organization (WHO), and WHO/Afro, in response to the registered challenges has worked modalities to be adopted by Member States as a way to addressing these concerns. Gradually, through the WHO strategy, TRM policy and legal framework has been adopted in most of the Member States in order to accommodate sustainable collaboration between THPs and the scientist/BHP. Research protocols on how to evaluate traditional medicines for safety and efficacy for priority diseases in Africa have been formulated. Creation of close working relationship between practitioners of both health care systems is strongly recommended so as to revamp trust among each other and help to access information and knowledge from both sides through appropriate modalities. In Tanzania, gaps that exist between THPs and scientists / BHP in health research have been addressed through recognition of THPs among stakeholders in the country's health sector as stipulated in the National Health Policy, the Policy and Act of TRM and CAM. Parallel to that, several research institutions in TRM collaborating with THPs are operating. Various programmed research projects in TRM that has involved THPs and other stakeholders are ongoing, aiming at complementing the two health care systems. This paper discusses global, regional and national perspectives of TRM development and efforts that have so far been directed towards bridging the gap between THPs and scientist/BHP in contemporary health research in Tanzania.
\end{abstract}

Keywords: Traditional, medicine, biomedical, scientist, practice, Tanzania

\section{Introduction}

Traditional health practitioner (THP), often referred to as traditional healer $(\mathrm{TH})$, is a person who is recognized by the community in which he lives as competent to provide health care by using plants, animal and mineral substances and other methods based on socio-cultural and religious background as well as the knowledge, attitudes and beliefs that are prevalent in the community regarding physical, mental and social well being and causation of diseases and disability (WHO, 1976). Traditional medicine (TRM), is defined as sum total of all knowledge and practical whether explicable or not used in diagnosis, prevention and elimination of physical, mental or social imbalance and relying exclusively on practical experience and observation handed down from generation to generation, whether verbal or in writing (WHO, 1976). In Tanzania, traditional and allopathic or conventional health care systems have coexisted since colonial times but operating in parallel circles although their main objective is to serve the same people. Colonialists, with their intension to rule Africa had to find a way to discourage all sort of activities which would have provided an opportunity for developing Africans. The whole process was calculated to ensure that the key features of African culture were captured and eroded and hence dis-empower the Africans. THPs used to play vital role in the doctrine of chiefdoms that existed during pre-colonial era. They were part of the ruling system assigned specifically to advise the chiefs on social and health wellbeing of their communities.

Colonial masters equipped with education and religious beliefs, brainwashed the African elites, which later led them to despise TRM and the role of traditional health care system that was embedded in the African traditional culture. This attitude allowed for penetration of foreign culture marking the beginning of mismatch between African traditional medicine and the orthodox or western-style medical care system and hence creating gaps between the two health care systems. The negative attitude towards traditional medicine and her practitioners created during the colonial rule is however, still lingering in many African scientists even today. Ironically, it is being acknowledged that African governments cannot provide health care to most of her people 
through conventional medicine based on the meagre budget allocated to the health sector. At certain times, the available modern health care facility has proved expensive to the majority poor populations. Partially, this explains why at least $80 \%$ of the population in the developing world relies on traditional medicine for their primary health care requirements (Farnsworth et al., 1985).

\section{Global strategies on traditional health practitioners and traditional medicine}

The role of THPs and expanded use of TRM in providing for health-well being of people is acknowledged worldwide. Besides providing for health care, TRM has economic potential through sale of medicinal plant products and in the protection of biodiversity and the environment through conservation of these plants. During the past two decades there has been noted a worldwide increase in utilization of TRM due to HIV/AIDS pandemic (UNAIDS 2000, 2002). Additionally, increase in microorganism resistance to the presently available drugs and hence resurgence of diseases like tuberculosis, malaria and several other HIV / AIDS-associated secondary infections suggests an upward trend in usage of TRM. Consequently, THPs are increasingly being involved in collaborative ventures with BHPs especially in sub-Saharan Africa where the diseases have escalated mortality and morbidity rates. This situation necessitates effective means for scientific validation of traditional medicines so as to either complement conventional health care system or to provide new drugs for better human health care.

WHO officially acknowledged the importance of traditional medicine and the role of THPs in the primary health care declaration of Alma-Ata in 1978. The value of THPs is endowed in their indigenous or traditional knowledge on the use of medicinal plants. In the African TRM, medicinal plants constitute $90 \%$ of materia medica used (WHO, 2005). Reliable information on the use of TRM therapies, particularly on medicinal plants, can help researchers and policy-makers to determine requirements in their national health systems as well as in clinical and scientific investigations pertaining to the efficacy and safety of traditional medicine. Moreover, such information is also needed in education and training of health care professionals in both modern and traditional systems of medicine. However, WHO acknowledges dearth of information and documentation on practices and utilization of different health care systems in the world today and especially for traditional medicine and for some of the complementary and alternative systems of medicine (WHO, 2002).

TRM has attained increased international popularity and demand but yet its efficacy, safety, quality and practice have not been thoroughly well established. This set back has become major concern for both health authorities and the public in general. Therefore, $\mathrm{WHO}$ has been strategically advocating worldwide for scientific validation of TRM with regard to efficacy, safety, quality and its practice. WHO 2002-05 strategy on TRM, was formulated specifically to address issues relating to policy; safety, efficacy and quality; access and its rational use globally (WHO, 2002). The overall aim is to enable TRM/CAM to play a far greater role in reducing excess mortality and morbidity, especially among impoverished populations. A plan of action and assignment of activities between WHO and its partners were outlined in the strategy.

On policy, the strategy insists integration of TRM/CAM with national health care systems, as appropriate, by developing and implementing national TRM/CAM policies and programmes. On safety, efficacy and quality - promote the safety, efficacy and quality of TRM/CAM by expanding the knowledge base on TRM/CAM, and by providing guidance on regulatory and quality assurance standards. For access — increase the availability and affordability of TRM/CAM, as appropriate, with an emphasis on access for poor populations. Lastly, concerning rational use - promote therapeutically sound use of appropriate TRM/CAM by THPs and consumers (WHO, 2002). In principle, WHO's role is to provide support to enable Member States to develop their own TRM/CAM and integrate it into their national health care policy and ensure appropriate, safe and effective use of TRM. WHO also seek the scientific community and the public to increase access to accurate information on TRM. The African Union (AU) declared 2001-2010 decade of African Traditional Medicine (Adeniji, 2002). The WHO/AFRO has also been promoting training involving THPs and THPs Associations and researchers 
on Intellectual Property Rights (IPRs) relating to traditional medicine. The aim is to empower the THP, especially on issues relating to accrued benefit sharing and royalties in collaborative research involving researchers, communities and development partners.

\section{Traditional medicine in Tanzania}

Recognition of THPs as health providers in Tanzania started during the German colonialists' era. The latter had always suspected THPs as threat to their ruling system and associated it with witchcraft and uncivilization. Nonetheless, some of the Christian missionaries accepted to use traditional remedies from THPs in their medical services and showed interest to study them (Kuelker, 2001). Recognizing the importance of herbal remedies, in 1895, German military doctors were officially advised to collect plant specimen and send them for scientific investigation in German. By1907, traditional medicine was incorporated in the health care system of the then "German East Africa" (Kuelker, 2001). THPs in Tanganyika were given certificates indicating their locations of practice and the illnesses they managed.

Despite the introduction of witchcraft Ordinance by the British colonialists in 1929, THPs were still allowed to practice in communities where respective traditional elders allowed them to do so (Kuelker, 2001). The practice of TRM was not recognized by the Government of Tanzania since independence until when the Ministry of Health issued a new Medical Practitioners and Dentists Ordinance of 1968. To date it is estimated that there are at least over 75,000 THPs in mainland Tanzania (Mshiu \& Chhabra, 1982). Presently, it is estimated that at least $60 \%$ of Tanzanians of the urban population and $80 \%$ of the rural population rely on TRM for their primary health care needs (Mahunnah, 2002). The government has demonstrated its political commitment to promoting TRM and at present the Tanzanian Health Policy recognizes TRM along with other CAM systems of health care (MoH, 1996). The political will of the government is further consolidated by the legislation passed by the Parliament in 2002 to establish the Traditional and Alternative Medicine Act.

\section{Gaps between traditional health practitioners and biomedical health practitioners}

In the course of practicing both TRM and Conventional Health Care systems, the major reason that causes parallelism is perhaps lack of common language on the perception of the causes and management of health problems. TRM does not regard man as a purely physical entity but also take into consideration the sociological environment whether living or dead (ancestors) and the intangible forces of the universe (spirits and gods) (WHO, 1976). TRM view disease/ illness as not merely the result of dysfunction of an organ due to material cause as perceived by the scientist / BHP, but it might also be due to an "intangible force". Consequently, treatment in TRM makes use not only of material substances but also of resources drawn from the "cosmic" or immaterial world (WHO, 1976).

TRM lacked political will and support as for colonialists it was considered a threat. Since, TRM is considered to be part of African culture, the approach used by foreigners to distort this health care system, created serious mistrust or attitudinal conflict between THPs and western or conventional medical practitioners. Furthermore, TRM lacked government commitment and consequently lacked policy and legal framework. The above gaps were inevitable because very little or sometimes nothing was known about TRM and there were no focused long-term plans in favour of its promotion. However, presently, majority of people understand better the importance of TRM and majority of world population use TRM and products from medicinal plants more than ever. It is only through formal collaboration between THPs and the scientists / BHPs that the existing gaps could be harmonized and hence provide for sustainable exploitation of TRM for better health for our people.

\section{Bridging the gaps}

The attempt made by the $\mathrm{MoH}$ to issue a new Medical Practitioners and Dentists Ordinance of 1968 was timely in terms of changing the mindset of especially Conventional Health Practitioners towards traditional medicine. The ordinance recognized the existence of THPs and their right to practice in Tanzania. The government's 
political commitment to promoting TRM is clearly spelled in the Tanzanian Health Policy that was formulated in 1996 and the Traditional and Alternative Medicine Policy and Act of 2000, and 2002, respectively, both of which recognize the role of THPs and TRM in the country's health care system (URT, 2000, 2002). Gradually, the government allowed for scientific research aimed at integrating TRM with allopathic health systems, through establishment of Traditional Medicine Research Unit at the University of Dar es Salaam in 1974. This unit was then elevated to an Institute of Traditional Medicine (ITM) in 1991. The University of Dar es Salaam (UDSM) departments of Botany, Chemistry, Marine Sciences and Microbiology are all routinely involved in teaching but also pursuing research in natural products. Within the Ministry of Health, a unit of TRM has been in place since 1989. The National Institute for Medical Research also joined the rally and started a department of traditional medicine research in 2002 to reinforce existing needs in research and development in TRM. In recognition of the value of indigenous knowledge, the Institute and the Ministry of Health, have worked together and finally came up with the TRM policy since 2000 (URT, 2000).

\section{Collaborative research between traditional and conventional health practitioners/scientist}

As potential health care stakeholders, THPs from several countries, including Tanzania, have shown their commitment towards fighting HIV / AIDS (Uiso et al., 2006; Kayombo et al., 2007). An ongoing collaborative endeavour between THPs and the scientists in Tanzania has been initiated aimed at evaluating safety, efficacy and toxicity of traditional herbal remedies used by THPs in the management of HIV / AIDS patients (Uiso et al., 2006, Kayombo et al., 2007). Careful analysis of the knowledge of THPs on HIV / AIDS captured through a series of seminars and structured questionnaires enabled the Institute to identify genuine and knowledgeable THPs on HIV / AIDS (Uiso et al., 2006). Formal agreement was reached through educational workshops and by involving legal partners from both parties a consensus was reached and in 2005 at least seven collaborating THPs signed Memorandum of Understanding with the ITM. Issues pertaining to ethical conduct, confidentiality, dissemination of research results and IPR were all considered within the signed MoUs. This approach helped the ITM to easily identify genuine healers for a disease like HIV / AIDS and has provided for mutual learning and trust for practitioners from both health care systems. As a result quite a number of remedies from several medicinal plants used by THPs in the management of HIV / AIDS have been documented. Analysis of study results further revealed that these remedies are safe, and efficacious against many HIV / AIDS-associated secondary infections, including diarrhoea, persistent cough, fever, oral thrush and skin problems (Z. Mbwambo, unpubl). Literature survey has shown good correlation between medicinal plants used by the collaborating THPs, which exhibit immunostimulant and antiviral activity and documented ethnobiomedical information for the same medicinal plants reported from other parts of the world. This direction encourages more research on Tanzanian medicinal plants for their potential in the management of HIV/ AIDS.

Another equally important project on TRM and HIV/AIDS being conducted in the spirit of mutual trust between BHPs is the Tanga AIDS working Group (TAWG) (UNAIDS, 2000). Reports by TAWG showed that 120 THPs were trained on AIDS counselling and care, promotion of condom use and behavioural change in the community. Practical field work including home-based care was part of the programme. Educational sessions for both BHPs and THPs were organized and above all patients referrals was done to and from both ends.

\section{Networking on medicinal plants and traditional medicine}

A network was launched in Arusha, Tanzania in November 2003. The collaborating partners include the Natural Chemotherapeutics Research Laboratory (Uganda), the Institute of Traditional Medicine of the Muhimbili University College of Health Sciences (Tanzania) and the National Museums of Kenya. This project seeks to set up a programmatic and collaborative platform that offers an opportunity for all stakeholders to share information and experience on their respective activities, harmonize their approaches and methodologies, and work together to develop 
projects that promote the conservation and sustainable, safe, and effective use of medicinal plants and herbal products, as well as the integration of TRM in public health services. To this end, the project focuses on: 1) assessment of research activities and policy frameworks in order to determine gaps and priorities both at national and sub-regional levels; 2 ) strengthening research capacity and harmonization of approaches and methodologies of researchers; 3) promoting the development of regional collaborative projects to attain critical masses for challenging/ "strategic" research projects and minimize duplication of efforts; 4) strengthening the capacity of traditional heath practitioner associations and their collaboration with other stakeholders (health workers, researchers, policy makers); 5) contributing to the development/streamlining of appropriate policy and frameworks; and 6) setting up processes to bridge gaps between researchers, communities and policy makers, and disseminate research results on TRM and medicinal plants for Eastern Africa.

A vision and strategy framework for conservation, management and utilization of biodiversity in East Africa has been developed. Development of Regional Project proposals in the areas of human health, eco-health, wealth creation and capacity building are at advanced stage of preparation. During the last 3 years, the Network carried out a number of activities to strengthen the capacity of THPs and THP Associations and their collaboration with other stakeholders. The Network organized workshops to develop regulations on materia medica for the operationalization of the Traditional and Alternative Medicine Act 2002. The THPs and THPs Associations, who are the custodians/ holders of indigenous medical knowledge of medicinal plants, were actively involved in the process of developing the regulations that will regulate their practice. Through the Network, the Traditional and Alternative Health Practice Council of Tanzania conducted a series of induction workshops on the operationalization of the 2002 Act in Tanzania mainland to ensure that THPs and their Associations understand and own the Act.

Similarly, a complimentary training tool/manual for BHPs is being developed by WHO/AFRO to orient them on aspects of traditional health practice. It is necessary to give orientation to the THPs in order for them to acquire some understanding as practitioners in the conventional health care system. The aim of the BHPs training programme is to increase their awareness on the important role being effectively played by the THPs in the promotion and provision of primary health care to the $80 \%$ of the African populations who regularly visit THPs for their health care needs, and encourage BHPs to be involved in TRM research activities.

\section{Conclusion}

Review on global, regional and country perspectives on TRM has revealed recognition and the role that THPs plays in the traditional health care system. The latter has had cultural acceptance in the majority of people and has the potential to solve people's health care problems both from developing and developed world even in this era of technological advances in conventional health care system. Furthermore, commercialization of medicinal and aromatic plants has been projected as a sustainable means of poverty alleviation and yet with additional value in terms of biodiversity and conservation purposes. Recognition of TRM worldwide have helped to reduce gaps that exist between the BHPs and THPs. Existing regulatory bodies, including WHO and AU encourage legal frameworks in place to safeguard, promote and ensure rational use of TRM through collaborative research and education for both practitioners of the two medical care systems. Additionally, policy and legal framework that should govern collaboration between the BHPs and THPs have to consider equitable sharing of benefits accruing from collaborative efforts and IPR. In Tanzania, the government has spearheaded recognition of THPs through Ministry of Health and their role as stakeholders in the Tanzania Health Policy. A legal framework has been put in place through the Traditional and Alternative Medicine Policy and Act of 2000, and 2002, respectively, and several research infrastructures in TRM established. THPs are increasingly organizing themselves in to associations and the ongoing collaborative research projects so far have significantly narrowed down the gaps between THPs and BHPs. 


\section{Recommendations and way forward}

In view of what has been disclosed above with regard to gaps between THPs and biomedical practitioners / researchers in contemporary health research in Tanzania, the following merit to be recommended. First, there is a need to consolidate and scale-up existing collaborations and training to offer sharing of experiences between THPs and biomedical practitioners / researchers using existing WHO/AFRO protocols and guidelines. Based on WHO recommendations, such undertakings should focus on priority diseases like HIV/AIDS, malaria, tuberculosis, diabetes, hypertension, etc. Secondly, there is a need to institutionalize a national platform or framework that will foster closer working relationship between the scientists/BHPs and THPs, THP associations, preferably a national THPs association. This body is expected to regulate among other things, issues of sharing of benefits and IPR. Lastly, there should be deliberate efforts directed towards increased awareness and advocacy on TRM to the public, policy-makers, researchers, THPs and end users through mass media, tailored radio/television programmes, flyers and other educational materials to ensure the two health care systems find a way to complement rather than competing with each other.

\section{References}

Adeniji, K.O. (2002) 2001-2010 Decade for African Traditional Medicine. Scientific, Technical and Research Commission, AU, Lagos.

Farnsworth, N. R., Akerele, O., Bingel, A. S., Soejarto, D. D. \& Zhengang, G. (1985) Medicinal plants in therapy. Bulletin of the World Health Organization 63, 965-981.

Gerard, B., Donna K., Rachel, K. \& Jacques, H. (2000) A regional task force on traditional medicine and AIDS. Lancet 355,1284.

Kayombo, E.J., Uiso, F.C., Mbwambo, Z.H., Mahunnah, R.L.A., Moshi, M.J. \& Mgonda, Y.H (2007) Experience of initiating collaboration of traditional healers in managing HIV and AIDS in Tanzania. Journal of Ethnobiology and Ethnomedicine 1, 3. Available from http:// www.ethnobiomed.com

Kuelker, K. (2001) The History of Health Care in
Tanzania. GTZ and National Museum of Tanzania, Dar es salaam. Pp. 2-4.

Mahunnah, R.L.A. (2002) Ethnobotany and conservation of medicinal plants in Africa. The way forward in the next decade. In: Adeniji, (ed.) Proceedings of the $15^{\text {th }}$ Meeting of the Inter-African Experts Committee on African Traditional Medicine and Medicinal Plants, Arusha, Tanzania, AU Lagos.

MoH (1996) National Health Care Policy. Ministry of Health, United Republic of Tanzania. Dar es Salaam, Government Printer.

Mshiu, E. N. \& Chhabra, S. C. (1982) Traditional Healers and Health Care Delivery in Tanzania. Tropical Doctor 12, 142-143.

Uiso, F.C., Kayombo, E.J., Mbwambo, Z.H., Mgonda, Y.H., Mahunnah, R.L.A. and Moshi, M.J. (2006) Traditional Healer's knowledge and implications to the management and control of HIV/AIDS in Arusha, Tanzania. Tanzania Health Research Bulletin 8, 95-100.

UNAIDS (2000) Collaboration with traditional healers in HIV/AIDS prevention and care in sub-Saharan Africa. A literature review, Geneva, Switzerland.

UNAIDS (2002) Ancient Remedies, New Disease: Involving Traditional Healers in Increasing Access to AIDS Care and Prevention in East Africa. UNAIDS/02.16E, Geneva, Switzerland.

URT (2000) National Traditional Healthcare Policy. United Republic of Tanzania. Dar es Salaam, Government Printer.

URT (2002) Traditional and Alternative Medicine Act No. 23 of 2002, United Republic of Tanzania. Dar es Salaam, Government Printer.

WHO (1976) African Traditional Medicine. Afro Technical Report series No. 1, World Health Organization, Regional Office for Africa, Brazzaville.

WHO (2000) General Guidelines for Methodologies on Research and Evaluation of Traditional Medicine, WHO /EDM/TRM/2000.1, Geneva, Switzerland.

WHO (2002) Traditional Medicine Strategy 200205, WHO/EDM/TRM2002.1, Geneva, Switzerland.

WHO (2005) Global Atlas of Traditional, Complementary and Alternative Medicine, Text volume, WHO, Centre for Health Development, Kobe, Japan. 